\title{
Engineering superfluidity in electron-hole double layers
}

\author{
Sergio Conti \\ Max-Planck-Institute for Mathematics in the Sciences, 04103 Leipzig, Germany \\ Giovanni Vignale \\ Department of Physics, University of Missouri, Columbia, Missouri 65211 \\ A. H. MacDonald \\ Department of Physics, Indiana University, Bloomington, Indiana 47405
}

(Received 29 December 1997)

\begin{abstract}
We show that band-structure effects are likely to prevent superfluidity in semiconductor electron-hole double-layer systems. We suggest the possibility that superfluidity could be realized by the application of uniaxial pressure perpendicular to the electron and hole layers. [S0163-1829(98)51712-2]
\end{abstract}

The possibility of realizing a superconducting condensate of electron-hole pairs in a system consisting of two spatially separated layers of electrons and holes was suggested some time ago. ${ }^{1}$ Only recently, however, has it become feasible ${ }^{2-5}$ to produce systems where the electrons and holes are close enough to interact strongly, and, at the same time, sufficiently isolated to inhibit optical recombination in nonequilibrium systems and tunneling between electron and hole bands. Since the overlap of the electron and hole wave functions in these systems can be made negligibly small, the joint motion of condensed electron-hole pairs turns out to be superfluid; antiparallel currents can flow in the two layers without dissipation. ${ }^{1,6}$ Although the electron-hole condensation temperature has been predicted to be in an accessible range, and signatures of its occurrence have been discussed in the literature, ${ }^{7,8}$ compelling evidence of a superfluid state is yet to appear. In this paper we propose a strategy for the realization of electron-hole superconductivity in double well systems. We point out that at high sufficiently densities, the anisotropy of the hole band in realistic wells is a major obstacle to the occurrence of superconductivity. We propose that the application of a moderate uniaxial stress ( $\sim 10 \mathrm{kbar})$ could reduce the anisotropy enough to permit the formation of a condensate.

Microscopic theories of superfluidity in electron-hole liquids have usually been developed in the framework of a simple mean field theory ${ }^{9}$ similar to the BCS theory of superconductivity. Recently, detailed numerical solutions of the BCS gap equation have been obtained for models of epitaxially grown double-layer structures. ${ }^{10-12} \mathrm{We}$ are interested in the high carrier density regime for which the underlying fermionic degrees of freedom of electrons and holes play an essential role in the pairing physics, and mean-field theory estimates of transition temperatures can be reliable. ${ }^{13}$ Indeed, recent variational ${ }^{14}$ and diffusion ${ }^{15}$ Monte Carlo calculations of the ground-state energy of an electron-hole double layer appear to qualitatively confirm BCS theory predictions for the dependence of the zero temperature gap on interlayer separation, provided that the attractive electronhole interaction is appropriately screened in estimating the BCS theory coupling constant. Although transition tempera- tures calculated with unscreened interactions (as high as 10 $\mathrm{K}$ with typical parameters) are expected to be overestimates, the naive expectation from these calculations is that the superfluid state should be within reach.

An aspect of the problem which is potentially important at high densities, and to which little attention has been paid thus far, is the influence of band structure on the BCS transition temperature. Previous calculations have assumed that electron and hole bands are both isotropic. ${ }^{16}$ Given this assumption, BCS theory predicts superfluidity for an arbitrary small value of the effective coupling constant $\lambda=N(0) V$, where $V$ is the characteristic magnitude of the attractive electron-hole interaction on the Fermi surface, $N(0)=m^{+} / 2 \pi \hbar^{2}$ is the density of pair states, and the effective mass $m^{+}$is related to the band masses by $1 / m^{+}=\left(1 / m^{(e)}+1 / m^{(h)}\right) / 2$. In reality, the band structures of experimentally relevant systems present substantial deviations from isotropy. In particular, the valence subbands of GaAs are strongly warped due to the interaction and avoided crossing of "light" and "heavy" hole bands illustrated in Fig. 1.

At densities of the order of $10^{11} \mathrm{~cm}^{-2}$ and higher, the variation of hole energies along the essentially circular electron Fermi line is $\sim 0.2 \mathrm{meV}$, larger than the value of $k_{B} T_{c}$ which would be expected if the hole bands were isotropic. Since the band anisotropy energy and the thermal energy have a similar deleterious influence on superfluidity, ${ }^{17}$ it is clear that the mismatch between electron and hole Fermi surfaces will have a dramatic impact on the critical temperature. As the coupling constant is decreased, a critical value of $\lambda$ will be reached where superfluidity is destroyed. It is therefore extremely important to assess whether or not superfluidity should be expected at any temperature in the systems fabricated with current technology or, if this is not the case, to propose a realistic procedure which can enhance pairing. This paper addresses precisely the above questions. We consider an AlAs/GaAs double-quantum-well system ${ }^{18}$ in which the GaAs wells have a width of $100 \AA$ and the separation between the layers is of order $100 \AA$ or larger. The densities of both electrons and holes are assumed to equal $2 \times 10^{11} \mathrm{~cm}^{-2}$. Under these conditions we find that the hole 


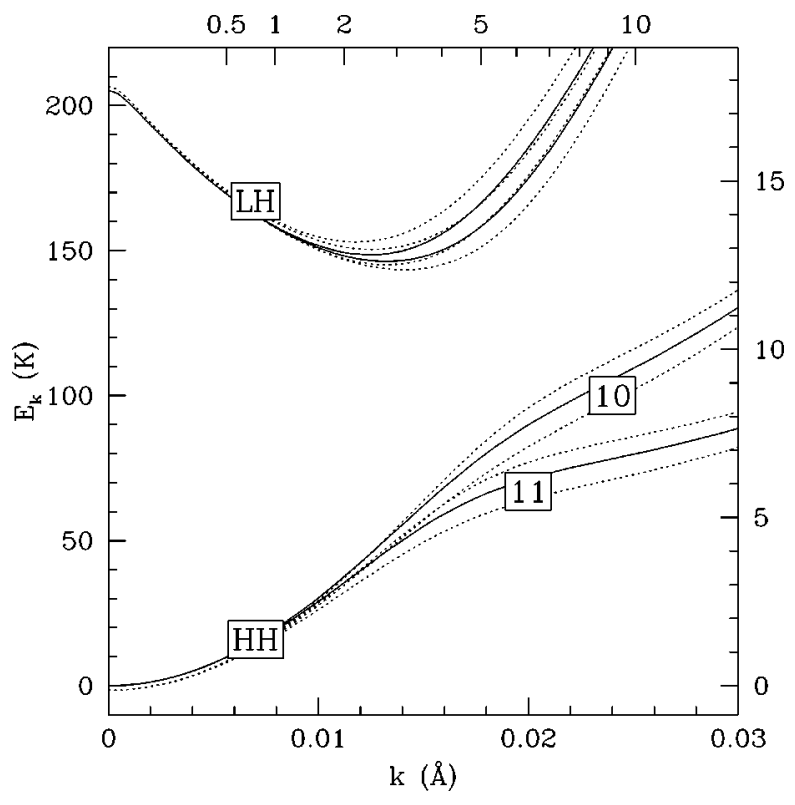

FIG. 1. Lowest heavy hole (HH) and the light hole (LH) subbands in the 11 and 10 directions, neglecting spin splitting (full curves) and including spin splitting due to an electric field $E=1 \mathrm{meV} / \AA$ (dotted curves). Energies in $\mathrm{K}$ and $\mathrm{meV}$ are given on the left and right axes, $k$ is in $\AA^{-1}$, and the top axis marks the isotropic Fermi wave vector $k_{F}=\sqrt{2 \pi n}$ for various densities $n$ (in units of $10^{11} \mathrm{~cm}^{-2}$ ).

band anisotropy effect is enough to destroy superfluidity, at least when the BCS coupling constant $\lambda$ is approximated using generalized random phase approximation (RPA) screening theory as discussed below.

The obvious route towards obtaining a finite $T_{c}$, via reduced electron-hole separation, is blocked by technological obstacles. Fortunately, the effect of the band anisotropy can be reduced by the application of a uniaxial stress. This is clearly illustrated in Figs. 2 and 3 which summarize the main results of this paper. We emphasize that the trends illustrated here are more reliable than the numerical results themselves. Although we cannot claim quantitative accuracy for the calculated $T_{c}$, it seems quite certain that the application of uniaxial stress will tend to increase or decrease $T_{c}$ as shown in Fig. 2. This information should therefore be valuable to experimenters trying to create optimal conditions for the observation of electron-hole superfluidity.

We now detail the calculations leading to the $T_{c}$ estimates summarized in Figs. 2 and 3. The four upper spin-orbit split $(j=3 / 2)$ valence band of a GaAs quantum well are calculated by diagonalizing the $4 \times 4$ Luttinger Hamiltonian ${ }^{19}$ in the envelope function approximation. ${ }^{20}$ The Hamiltonian has the form

$$
H\left(\vec{k}, z, \frac{\partial}{\partial z}\right)=H_{\text {bulk }}\left(k_{x}, k_{y}, k_{z} \rightarrow-i \frac{\partial}{\partial z}\right)+V(z),
$$

where $\vec{k}=\left(k_{x}, k_{y}\right)$ is the wave vector in the plane of the quantum well, $z$ is the perpendicular direction, and $V(z)$ is the confinement potential. The bulk Hamiltonian is

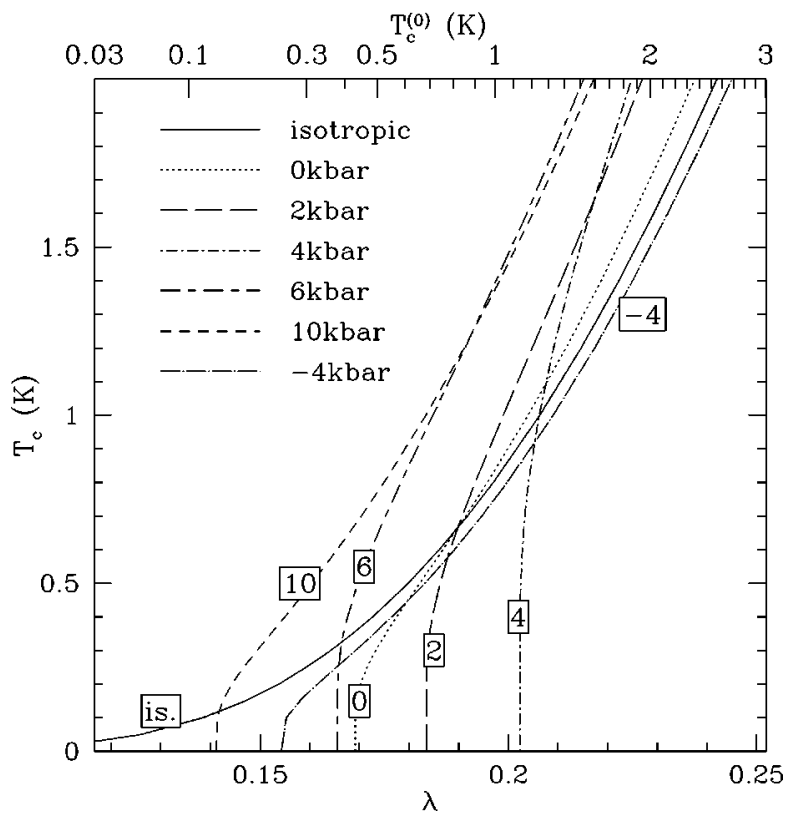

FIG. 2. Critical temperature as a function of $\lambda$ at various values of the applied uniaxial pressure $P$ (expressed in kbar). The curve labelled "is" was calculated using an isotropic approximation to the $P=0$ hole bands. The top axis reports $T_{c}^{(0)}=1.14 e^{-1 / \lambda}$.

$$
H_{\text {bulk }}\left(k_{x}, k_{y}, k_{z}\right)=\left(\begin{array}{cccc}
a_{+} & b & c & 0 \\
b^{*} & a_{-} & 0 & c \\
c^{*} & 0 & a_{-} & -b \\
0 & c^{*} & -b^{*} & a_{+}
\end{array}\right),
$$

where

$$
\begin{aligned}
a_{ \pm}= & \frac{1}{2 m_{0}}\left(\gamma_{1} \pm \gamma_{2}\right)\left(k_{x}^{2}+k_{y}^{2}\right)+\frac{1}{2 m_{0}}\left(\gamma_{1} \mp 2 \gamma_{2}\right) k_{z}^{2} \\
& \mp \frac{2 X}{3} D_{u}\left(S_{11}-S_{12}\right),
\end{aligned}
$$

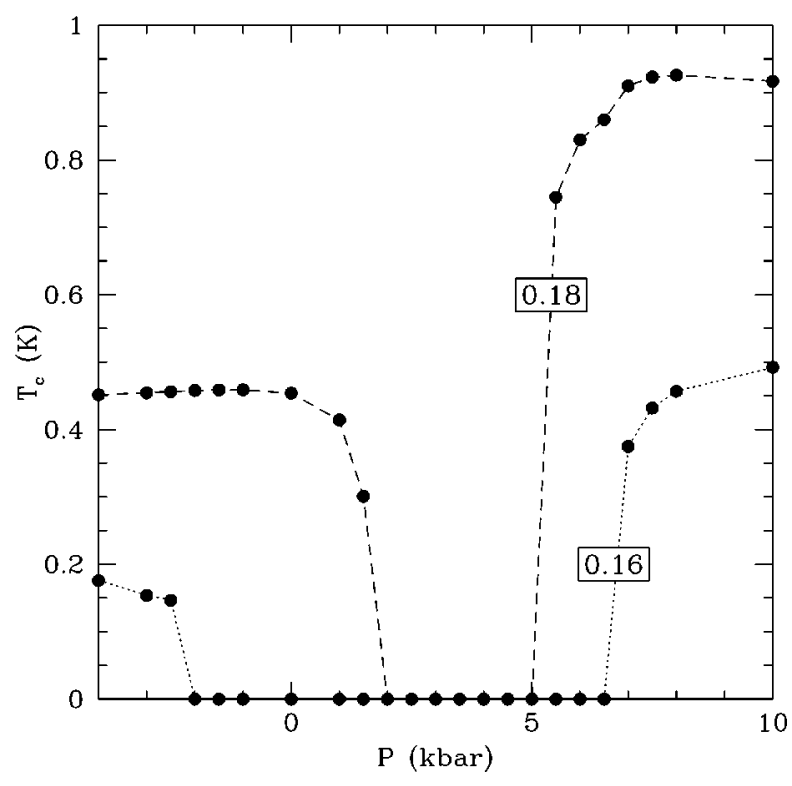

FIG. 3. Critical temperature as a function of applied pressure $P$ for two different values of $\lambda$. 


$$
\begin{gathered}
b=-i \sqrt{3} \gamma_{3}\left(k_{x}-i k_{y}\right) k_{z} / m_{0}, \\
c=\frac{\sqrt{3}}{2 m_{0}}\left[\gamma_{2}\left(k_{x}^{2}-k_{y}^{2}\right)-2 i \gamma_{3} k_{x} k_{y}\right],
\end{gathered}
$$

the Luttinger parameter for GaAs are ${ }^{19} \gamma_{1}=6.85, \gamma_{2}=2.10$, and $\gamma_{3}=2.9$, and $m_{0}$ is the bare electron mass. The parameter $X$ represents an externally applied uniaxial pressure in the growth direction. For GaAs the elastic compliance constants $S_{11}=1.17 \times 10^{-3} \mathrm{kbar}^{-1}$ and $S_{12}=-0.37 \times 10^{-3}$ $\operatorname{kbar}^{-1}$, and $D_{u}=-2.5 \mathrm{eV}{ }^{21}$ The presence of the thin AlAs barrier between the GaAs substrate and the GaAs quantum well can be neglected in the study of elastic properties. We approximate $V(z)$ by a "square well" potential $[V(z)=0$ in the well and $V(z)=0.6 \mathrm{eV}$ in the barrier].

The band structure can be obtained following the method of Andreani et $a .^{21}$ In the absence of applied stress one obtains the doubly degenerate subbands shown in Fig. 1. Neglecting a narrow pressure range around $4 \mathrm{kbar}$, at the densities of interest only the lowest energy subband is occupied. However, interaction between subbands is very strong, and causes considerable nonparabolicity and anisotropy. The double degeneracy is a consequence of time reversal invariance and inversion symmetry with respect to the plane of the well: it is therefore lifted (at $\vec{k} \neq 0$ ) by any potential $V(z)$ which does not possess inversion symmetry.

Given the band structure, we can estimate the superconducting gap by solving the BCS gap equation,

$$
\Delta_{k}=\sum_{k^{\prime}} V\left(\vec{k}-\vec{k}^{\prime}\right) \frac{\Delta_{k^{\prime}}}{2 E_{k^{\prime}}}\left[1-f\left(E_{k^{\prime},+}\right)-f\left(E_{k^{\prime},-}\right)\right] .
$$

Here $\Delta_{k}$ is the "gap" function, and $E_{k, \pm}$ are the BCS theory quasiparticle energies of the superconductor given, for the case of unequal electron and hole band dispersions, by $E_{k, \pm}=E_{k} \pm \eta_{k}, \quad E_{k}=\sqrt{\epsilon_{k}^{2}+\Delta_{k}^{2}}, \quad \epsilon_{k}=\left(\epsilon_{k}^{(e)}+\epsilon_{k}^{(h)}\right) / 2, \quad$ and $\eta_{k}=\left(\epsilon_{k}^{(e)}-\epsilon_{k}^{(h)}\right) / 2$. $\epsilon_{k}^{(e)}$ and $\epsilon_{k}^{(h)}$ are the band energies of the lowest conduction and valence bands (the former taken to be parabolic with effective mass $m^{(e)}=0.067 m_{0}$ ) relative to the electron and hole chemical potentials respectively, and $V\left(\vec{k}-\vec{k}^{\prime}\right)$ is the effective electron-hole interaction potential. (Notice that $\Delta_{k}$ does not represent the minimum excitation energy of the superconductor.) Equation (6) is the meanfield-theory gap equation for the spin-unpolarized electronhole pairs of the expected ${ }^{9}$ condensed state.

To obtain our estimates we follow BCS theory custom by replacing the attractive interaction $V\left(\vec{k}-\vec{k}^{\prime}\right)$ by a constant matrix element $V$, which presumably represents an appropriate average of the true interaction over the relevant wavevector range. We also restrict the momentum summation so that only states with band energies within a cutoff energy $\Omega_{c}$ of the Fermi surface are included, where $\Omega_{c}=\left(4 \pi e^{2} n k_{F} / m^{+} \epsilon_{0}\right)^{1 / 2}$ is the plasma frequency at the Fermi wave vector.

We see in Fig. 2 that the main effect of the anisotropy is to introduce a minimum value of $\lambda=N(0) V$ below which there is no superconductivity. ${ }^{22}$ The origin of the minimum is obvious; the familiar logarithmic divergence coming from the region of small $E_{k}$ in the sum of Eq. (6) is suppressed at low temperature by the thermal factor $1-f\left(E_{k,+}\right)-f\left(E_{k,-}\right)$ since either $E_{k,+}$ or $E_{k,-}$ is negative for small $\left|E_{k}\right|$. The right-hand side of Eq. (6) is finite and no solution other than $\Delta=0$ can be found, even for $T \rightarrow 0$, if $V$ is too small. Upon application of a compressive uniaxial stress the minimum value at first increases, because the heavy-hole and light-hole bands are squeezed towards each other, increasing the anisotropy. At a pressure of about 4 kbar the two bands cross. Further pressure increases make the valence bands increasingly isotropic: hence, the minimum $\lambda$ decreases, and the transition temperature increases. A similar effect can also be obtained by applying a tensile uniaxial stress, or, equivalently, by applying an isotropic compressional strain in the plane of the wells.

An accurate calculation of $\lambda$ is difficult. For example, use of the unscreened interaction at the Fermi wave vector $V=v^{\text {bare }}\left(k_{F}\right)$ gives $\lambda \simeq 0.36$ and $T_{c} \simeq 10 \mathrm{~K}$ for $d=100 \AA$. Similar estimates result from detailed $T_{c}$ calculations which do not account for screening. ${ }^{11,10}$ At such a large value of $\lambda$, band structure effects would be irrelevant. However, screening is expected to reduce the coupling strength considerably. An improved estimate of $\lambda$ can be obtained from the longwavelength limit of the screened electron-hole interaction; ${ }^{23}$

$$
\lambda \approx N(0) V_{e h}(k=0)=\frac{a_{B}^{(e)} a_{B}^{(h)} / 4 a_{B}^{+}}{a_{B}^{+}+2 d-\xi_{d}}+\frac{\xi_{\mathrm{eh}}}{a_{B}^{+}} .
$$

[Here $a_{B}^{+}=\left(a_{B}^{(e)}+a_{B}^{(h)}\right) / 2 \sim 80 \AA$ is the average effective Bohr radius of GaAs, $\xi_{d}=\xi_{e e}+\xi_{h h}-2 \xi_{e h}$, $\xi_{i j}=\lim _{k \rightarrow 0} G_{i j}(k) / k$, and $G_{i j}(k)$ are static local field corrections of the Singwi, Tosi, Land, and Sjölander (STLS) (Refs. 24 and 25) type. For the purpose of estimating $\lambda$ we use parabolic bands.] The RPA, for which $\xi_{i j}=0$, gives $\lambda \sim 0.07$. Using STLS to compute $\xi$ we obtain $\lambda \sim 0.1$. The same result is obtained by neglecting $\xi_{\text {eh }}$ and evaluating $\xi_{e e}, \xi_{h h}$ from the single-layer equation of state $^{26}$ via the compressibility sum rule. With these values of $\lambda$, band structure effects would destroy superfluidity at all reasonable pressures. However, as illustrated in Fig. 3, at intermediate values of $\lambda$ a phase transition to the superfluid state can be induced by the application of a moderate pressure. Similar results are obtained for InAs-GaSb quantum wells.

The above calculations are for $100 \AA$-wide wells, whereas interactions can be strengthened and band anisotropies weakened by making the wells narrower favoring a superfluid state. However, for narrower wells the carrier densities tend to have stronger spatial inhomogeneities. Pairing requires the densities in the two wells to be locally equal; a BCS state will occur only if the density fluctuation $\delta n / n$ is smaller than $1 / k_{F} \xi \simeq 3 \times 10^{-2}$, where $\xi$ is the coherence length. Another important requirement is that disorder scattering, which will not typically be correlated in the two layers, be weak. The scattering time $\tau$ should satisfy $\hbar / \tau<\Delta$, which for a mobility $\mu=10^{6} \mathrm{~cm}^{2} / \mathrm{Vs}$ gives $\Delta / k_{B}>0.2 \mathrm{~K}$.

In closing we discuss the effect of the lifting of degeneracy of the hole subbands when the self-consistent 
quantum-well confinement potential does not have an inversion center. In Fig. 1 we show the effect of an electric field $E \simeq 1 \mathrm{meV} / \AA$. The field combines with spin-orbit coupling at the atomic level to split the $j=+3 / 2$ and $j=-3 / 2$ heavyhole subbands at finite wave vector. For a sample where nonvanishing equilibrium electron and hole densities are realized via an external electric field, $E \sim 10 \mathrm{meV} / \AA$ and the splitting of the Kramers degeneracy of the hole bands will be large. In this circumstance only one of the two split subbands will have the same density as the electron layer and therefore have a good chance to condense. In general, the spin structure of the condensate will be quite sensitive to details of the band structure, adding to the richness of the phenomenology to be studied if this state can be achieved.

This work was supported in part by the National Science Foundation under Grants No. DMR-9706788 and No. DMR9714055. We gratefully acknowledge useful discussions with Silvano De Franceschi and J. C. Maan.
${ }^{1}$ Yu. E. Lozovik and V. I. Yudson, Pis'ma Zh. Éksp. Teor. Fiz. 22, 556 (1975) [JETP Lett. 22, 271 (1975)]; Zh. Éksp. Teor. Fiz. 71, 738 (1976) [Sov. Phys. JETP 44, 389 (1976)]; S. I. Shevchenko, Fiz. Nizk. Temp. 2, 505 (1976) [Sov. J. Low Temp. Phys. 2, 251 (1976)].

${ }^{2}$ T. Fukuzawa, E. E. Mendez, and J. M. Hong, Phys. Rev. Lett. 64, 3066 (1990).

${ }^{3}$ J. A. Kash, M. Zachau, E. E. Mendez, J. M. Hong, and T. Fukuzawa, Phys. Rev. Lett. 66, 2247 (1991).

${ }^{4}$ U. Sivan, P. M. Solomon, and H. Shtrikman, Phys. Rev. Lett. 68, 1196 (1992).

${ }^{5}$ B. E. Kane, J. P. Eisenstein, W. Wegscheider, L. N. Pfeiffer, and K. W. West, Appl. Phys. Lett. 65, 3266 (1994).

${ }^{6}$ The absence of a significant electron-hole overlap is crucial for the occurrence of superconductivity [Ref. 1 and S. I. Shevchenko, Phys. Rev. Lett. 72, 3242 (1994)]. Otherwise condensation would lead to the so-called "excitonic insulator" studied extensively during the sixties $\{$ L. V. Keldysh and Yu. V. Kopaev, Fiz. Tverd. Tela (Leningrad) 6, 2791 (1964) [Sov. Phys. Solid State 6, 2219 (1965)]; D. Jerome, T. M. Rice, and W. Kohn, Phys. Rev. 158, 462 (1967)\}.

${ }^{7}$ G. Vignale and A. H. MacDonald, Phys. Rev. Lett. 76, 2786 (1996).

${ }^{8}$ Yu E. Lozovik and V. I. Yudson, Fiz. Tverd Tela 18, 1962 (1976) [Sov. Phys. Solid State 18, 1142 (1976)].

${ }^{9}$ C. Comte and P. Nozières, J. Phys. (Paris), Colloq. 43, 1069 (1982).

${ }^{10}$ Y. Naveh and B. Laikhtman, Phys. Rev. Lett. 77, 900 (1996).

${ }^{11}$ Xuejun Zhu, P. B. Littlewood, Mark S. Hybertsen, and T. M. Rice, Phys. Rev. Lett. 74, 1633 (1995).

${ }^{12}$ Z. W. Gortel and Leszek Swierkowski, Surf. Sci. 361/2, 146 (1996).

${ }^{13}$ The superfluid transition in two dimensions is expected to be a Kosterlitz-Thouless transition for which the transition temperature and the superfluid stiffness are related by $k_{B} T_{c}=\pi \rho_{s} / 2$. For equal electron and hole masses, BCS theory in two dimensions gives $\rho_{s} \approx\left(1-T / T_{c}^{M F}\right) E_{F} / 4 \pi$. Since the mean-field transition temperatures obtained below are typically $\sim E_{F} / 100 k_{B}$ or smaller, the Kosterlitz-Thouless $T_{c}$ is only slightly smaller than $T_{c}^{M F}$.

${ }^{14}$ A. Chan, Ph.D. thesis, Indiana University, 1996.

${ }^{15}$ F. Rapisarda and G. Senatore (unpublished).

${ }^{16}$ Following earlier work we consider the favorable case of equal electron and hole densities, assuming that this condition can be achieved by adjusting gate voltages.

${ }^{17}$ J. Zittartz, Phys. Rev. 162, 752 (1967).

${ }^{18}$ At least two other types of electron-hole double-layer systems are of current interest. The systems studied in Ref. 10 are based on the type-II heterojunction between GaSb and InAs for which the materials technology is less well developed than in GaAs/AlAs systems. Optically generated electron-hole double layers can also be created in a single GaAs/AlAs quantum well. In this case the electrons and holes are not in equilibrium, so that optical recombination plays a role, and hybridization of conduction and valence bands can become a serious issue. The main lines of our discussion would apply equally well to these other cases.

${ }^{19}$ Weng W. Chow, Stephan W. Koch, and Murray Sargent III, Semiconductor Laser Physics (Springer-Verlag, Berlin, 1994), Chap. 6.

${ }^{20}$ M. Altarelli, in Heterojunctions and Semiconductor Superlattices, edited by G. Allan et al. (Springer-Verlag, Berlin, 1985).

${ }^{21}$ L. C. Andreani, A. Pasquarello, and F. Bassani, Phys. Rev. B 36, 5887 (1987).

${ }^{22}$ The minimum $\lambda$ can be estimated from $\Omega_{c} e^{-1 / \lambda_{\min }} \sim k_{F} \delta k_{F} / m^{+}$, where $\delta k_{F}$ is the root-mean-square Fermi wave-vector difference between electrons and holes.

${ }^{23}$ G. Vignale and K. S. Singwi, Phys. Rev. B 32, 2156 (1985).

${ }^{24}$ K. S. Singwi, M. P. Tosi, R. H. Land, and A. Sjölander, Phys. Rev. 176, 589 (1968).

${ }^{25}$ Lian Zheng and A. H. MacDonald, Phys. Rev. B 49, 5522 (1994).

${ }^{26}$ F. Rapisarda and G. Senatore, Aust. J. Phys. 49, 161 (1996). 